\title{
Molecular Anapole Moments
}

\author{
A. Ceulemans and L.F. Chibotaru* \\ Department of Chemistry, University of Leuven, Celestijnenlaan 200F, B-3001 Leuven, Belgium \\ P. W. Fowler \\ Department of Chemistry, University of Exeter, Stocker Road, Exeter, United Kingdom EX4 4QD
}

(Received 18 July 1997)

\begin{abstract}
A quantum mechanical formalism for molecular anapole moments is developed. London-model calculations show that toroidal carbon frameworks support both permanent and induced anapole moments arising from $\pi$-electronic, through-bond currents; dipolar flow in a chiral toroid implies an anapolar component. [S0031-9007(98)05425-8]
\end{abstract}

PACS numbers: $33.15 . \mathrm{Kr}, 36.90 .+\mathrm{f}$

The recent observation by Liu et al. of circular ropes in scanning force micrographs of carbon nanotube materials [1] heralds the isolation of perfect molecular toroids. Such molecules are expected to exhibit several unusual properties directly related to their special topology. A toroidal network of bonds has two topological flow invariants: a "latitudinal" circulation around the central hole and a "longitudinal" circulation linking the inside and outside of the toroidal ring $[2,3]$. This second flow has the symmetry of an electric dipole and gives rise to an anapole moment. The existence of nuclear anapole moments, first predicted by Zel'dovich [4], has recently been confirmed by the measurement of parity nonconservation in the $6 S \rightarrow 7 S$ transition of Cs [5]. These effects may be enhanced by the anisotropy of the molecular environment [6]. The effects of parity nonconservation in atoms and molecules are discussed in the book by Khriplovich [7] and widely reviewed [8,9]. Paramagnetic atoms and molecules also can exhibit an anapole moment of nonparity violating electromagnetic origin in the presence of electric fields [10,11]. However, a genuinely molecular manifestation of the anapole moment has had to await molecular toroids. In the present study we develop the necessary quantum mechanical formalism and perform some model calculations on carbon toroids.

The molecular anapole moment can be derived from the multipole expansion of the electromagnetic interaction Hamiltonian, $\hat{V}$, for a molecule in an external magnetic field, B. In Raab's formalism [12] the second-order magnetic moment operators including both spin and orbital contributions are obtained as

$$
\hat{M}_{\alpha \beta}=-2 \frac{\partial \hat{V}}{\partial B_{0 \beta \alpha}},
$$

in which $B_{0 \beta \alpha}=\left(\nabla_{\beta} B_{\alpha}\right)_{0}$ at a given origin zero. The antisymmetric combination of these moments with an appropriate multiplication factor yields the anapole moment operator $\hat{\mathbf{a}}$. Hence for the component along the axis of the toroid one has

$$
\hat{a}_{z}=-\frac{1}{2}\left(\hat{M}_{x y}-\hat{M}_{y x}\right) .
$$

The anapole moment operator defined in this way describes the interaction of the system with the rotor of the external field. Indeed defining $\mathbf{B}^{\prime}=\nabla \times \mathbf{B}$, one immediately obtains

$$
\hat{\mathbf{a}}=-2 \nabla_{\mathbf{B}^{\prime}} \hat{V} .
$$

The expectation value of $\mathbf{a}$ is then found by applying the Hellmann-Feynman theorem

$$
\langle\hat{\mathbf{a}}\rangle=-2 \nabla_{\mathbf{B}^{\prime}} E,
$$

where $E$ is the electronic energy in an external magnetic field. In principle, in order to induce an anapole moment in a toroidal molecule, one would need a circular magnetic field that follows the loop of the torus. However, in toroidal molecules of chiral symmetry, even a uniform magnetic field along the principal axis of revolution may induce an anapole response. Indeed permanent magnetic moments in open-shell molecules of this type also have an anapole component. These points will now be illustrated by model calculations.

Two model toroidal carbon cages were investigated. Each has 120 atoms, 10 pentagonal, 60 hexagonal, and 10 heptagonal faces. The structure shown in Fig. 1(a) has $D_{5 d}$ symmetry, while that in 1 (b) has only $D_{5}$ symmetry. Both were optimized by molecular mechanics, which gave structures with a small $(\leq 3 \%)$ spread of bond lengths; all

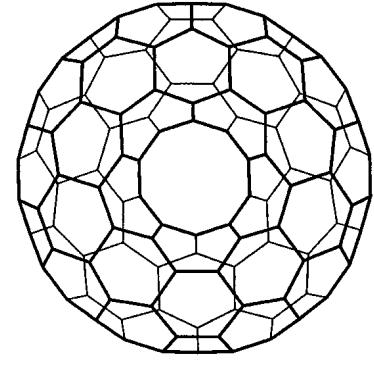

(a)

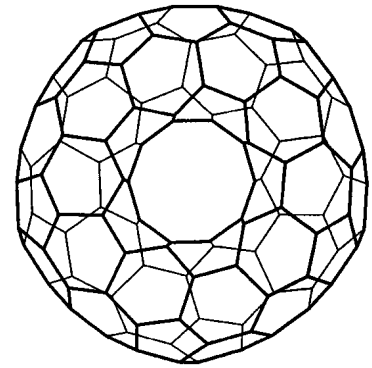

(b)
FIG. 1. Model 120-atom carbon toroids: (a) an achiral $D_{5 d}$, and (b) a chiral $D_{5}$ structure.

(C) 1998 The American Physical Society

1861 
coordinates were scaled to a mean bond length of $1.4 \AA$ for comparison with previous magnetic calculations on fullerenes [13]. The $D_{5 d}$ structure has been used as a model toroidal fullerene analog in earlier mathematical and computational studies $[2,14]$. The $D_{5}$ isomer is obtained from it by five concerted Stone-Wales rotations [15] of pentagon pairs (see Fig. 2). In the crude Hückel approximation the $D_{5 d}$ cage has an $e^{2}$ open shell as a neutral, but the $D_{5}$ isomer has a properly closed $e^{4}$ shell.

Magnetic moments were calculated using the London method [16] in conjunction with the Hückel theory (which considers $\pi$ electrons only). In this approach the site orbitals are transformed by a gauge factor incorporating a vector potential that generates the external field. The Hückel matrix elements are consequently approximated as [17]

$$
\begin{aligned}
h_{k l} & =\beta_{k l} \exp \left(2 \pi i f_{k l}\right), \\
\text { with } f_{k l} & =\frac{e}{2 h c}\left(\mathbf{A}_{k}-\mathbf{A}_{l}\right) \cdot\left(\mathbf{R}_{k}+\mathbf{R}_{l}\right) .
\end{aligned}
$$

Here $\beta_{k l}$ is the transfer integral between $\pi$ orbitals on sites $k$ and $l$. $\mathbf{A}_{k}$ denotes the value of the vector potential at the position $\mathbf{R}_{k}$ of site $k$. The ground state $\pi$ energy simply corresponds to the sum of the eigenvalues of this (complex) matrix for occupied levels. For a uniform rotor field $\mathbf{B}^{\prime}$ the vector potential can be expressed as

$$
\mathbf{A}(\mathbf{r})=\frac{1}{6} \mathbf{r} \times\left(\mathbf{r} \times \mathbf{B}^{\prime}\right) .
$$

$B^{\prime}$ was aligned here along the $C_{5}$ axis. The first derivative of $E$ with respect to $B^{\prime}$ yields the anapole moment, $a_{z}=-2 \partial E / \partial B^{\prime}$, while the second derivative corresponds

\section{(a)}
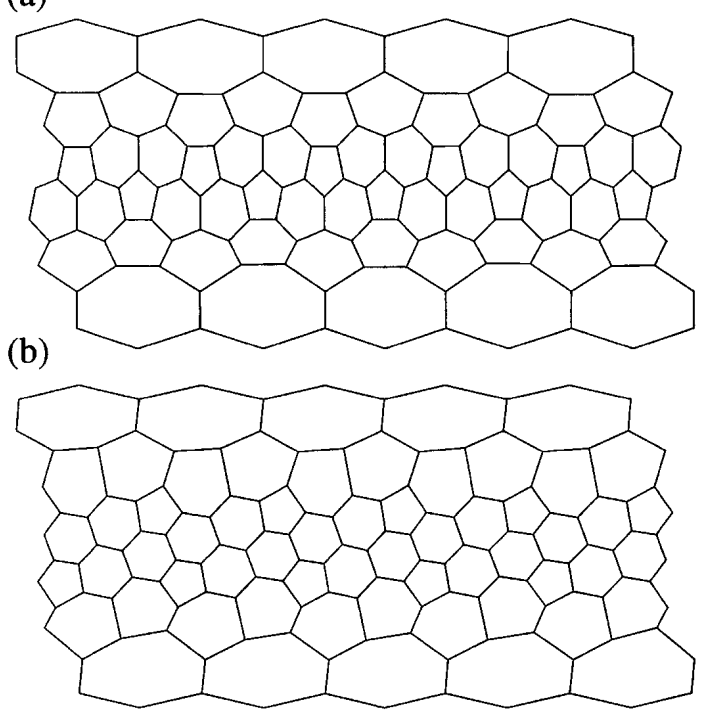

FIG. 2. Projections of the two toroidal polyhedral networks (a) and (b) obtained by unpeeling the toroidal surface. Top and bottom edges of the patch represent the inner equatorial circuit of the torus, the outer equatorial circuit running horizontally across the middle of the patch. Gluing the patch first top to bottom as a tube, then side to side, recovers the 3D torus. Bond lengths are distorted by the equal-angle projection. to the anapole-type susceptibility, $A_{\|}=-2 \partial^{2} /\left(\partial B^{\prime}\right)^{2}$, just as derivatives with respect to a homogeneous field yield the dipole moment, $m_{z}=-\partial E / \partial B$, and the magnetic susceptibility, $\chi_{\|}=-\partial^{2} E / \partial B^{2}$. In the chiral toroid there is additionally a symmetry-allowed cross term of the type $-\left(\partial^{2} E / \partial B^{\prime} \partial B\right)$ that will be denoted as $M_{\|}$. The results of the calculations are displayed in Table I. In the units used here, Cartesian coordinates are in bohr, energies in units of a single Hückel parameter $|\beta|$ for all bonds, $B$ in $\hbar c / e a_{0}^{2}$, and $B^{\prime}$ in $\hbar c / e a_{0}^{3}$. In this convention benzene with a bond length of $1.4 \AA$ has a diamagnetic $\pi$-electron susceptibility $\chi_{\|}=-73.485$, corresponding to the scaled value of -1.5 given by Elser and Haddon [18].

As already pointed out by Haddon [19], toroids can be expected to have large magnetic dipole susceptibilities. But in the present (spin-free) treatment they are also seen to have large induced anapole moments that may be of either sign. Half-filled open-shell and closed-shell cages carry no orbital moment, in contrast to $e^{1}$ and $e^{3}$ configurations. For the $D_{5 d} e^{1}$ cation the anapole moment vanishes because of parity, but in the chiral $D_{5} e^{3}$ cation an anapole moment is found at zero field (Table I). These symmetry selection rules can easily be generalized. In any system with a principal axis of symmetry, the antisymmetrized square of a degenerate irreducible representation always transforms as the parallel component of the magnetic dipole operator, whereas the anapole moment transforms as an electric dipole operator. The anapole moment will therefore be allowed only in the absence of improper symmetry elements. In contrast, nonzero anapole susceptibilities are compatible with all point groups, but will require toroidal or higher topology for significant throughbond effects. Electron spin will make contributions to permanent magnetic moments in open-shell states.

For a further analysis of these results we now turn to the description of the current densities. For a homogeneous

TABLE I. Calculated dipole and anapole magnetic properties for the two model carbon tori of Fig. 1. ${ }^{a}$

\begin{tabular}{lrrrr}
\hline \hline & \multicolumn{2}{c}{$D_{5 d}$} & \multicolumn{2}{c}{$D_{5}$} \\
& neutral $e^{2}$ & cation $e^{1}$ & neutral $e^{4}$ & cation $e^{3}$ \\
\hline$\chi_{\|}$ & -2200 & -1890 & -1130 & -1180 \\
$A_{\|}$ & 5420 & 8000 & -12600 & -9350 \\
$m_{z}$ & 0 & 2.74 & 0 & 4.86 \\
$a_{z}$ & 0 & 0 & 0 & 13.9 \\
$M_{\|}$ & 0 & 0 & -265 & -380 \\
\hline \hline
\end{tabular}

${ }^{a}$ All properties are defined as derivatives of the $\pi$-electronic energy (see text) and are quoted here in units of $|\beta|(e / \hbar c) a_{0}^{n}$ where $n=2$ and 3 for $m, a$, and of $|\beta|(e / \hbar c)^{2} a_{0}^{n}$ where $n=4$, 5 , and 6 for $\chi, M$, and $A$, and $\beta$ is the Hückel bond parameter; $\chi$ and $m$ are independent of origin. The origin for $A, a$, and $M$ is the center of mass. In Hückel theory $\beta$ is the Hamiltonian integral between basis functions on neighboring atoms, giving the scale of orbital energies, and thus in the purely topological London model used here appears as part of the unit for each response property. An empirical value of $2.66 \mathrm{eV}$ is often used in converting magnetic properties such as $\chi$ to SI units [22]. 
magnetic field, $\mathbf{A}=-\frac{1}{2} \mathbf{r} \times \mathbf{B}$, the phase factor of the Hückel-London parametrization may be written

$$
f_{k l}=\frac{e}{h c} \mathbf{B} \cdot \mathbf{S}_{k l},
$$

where $\mathbf{S}_{k l}=\frac{1}{2}\left(\mathbf{R}_{k} \times \mathbf{R}_{l}\right)$ is the normal to the triangle spanned by the vectors $\mathbf{R}_{k}$ and $\mathbf{R}_{l}$. The quantity $f_{k l}$ thus corresponds to the magnetic flux $\mathbf{B}$ across this triangle. The corresponding through-bond current may then be obtained as in classical magnetism by differentiating the total energy with respect to the magnetic flux

$$
J_{k l}=-\frac{e}{h} \frac{\partial E}{\partial f_{k l}} .
$$

This expression is also valid in the case of a nonhomogeneous magnetic field, with $f_{k l}$ defined as in (5). By the Hellmann-Feynman theorem this current contribution may be resolved into a sum over occupied molecular orbitals

$$
J_{k l}=\frac{2 e \beta_{k l}}{\hbar} \sum_{\mu}^{o c c} \operatorname{Im}\left[c_{\mu k}^{*} c_{\mu l} \exp \left(2 \pi i f_{k l}\right)\right] .
$$

Here $c_{\mu k}$ is the coefficient of the orbital on site $k$ in the $\mu$ th occupied molecular orbital. This result can also be derived from a momentum operator formalism. We start from a standard expression for the nondiagonal matrix element of the current density operator [20]

$$
\hat{J}_{k l}=\frac{i e \hbar}{2 m_{e}}\left[\left(\nabla \phi_{k}^{*}\right) \phi_{l}-\phi_{k}^{*} \nabla \phi_{l}\right],
$$

where $\phi_{k}$ and $\phi_{l}$ are atomic $\pi$ orbitals in a given pair of atoms and $m_{e}$ is the electronic mass. Equation (10) does not contain the contribution from the vector potential, which is in line with approximations made within the London theory $[16,17]$. To obtain the total current we integrate the current density over a surface cutting the bond. This surface integral can be obtained by taking a volume integral divided by the length of the bond. Thus the bond current operator in second quantization has the form

$$
\hat{\mathbf{J}}_{k l}=\frac{e}{m_{e}} \frac{1}{\left|\mathbf{R}_{k}-\mathbf{R}_{l}\right|}\left[a_{k}^{\dagger} a_{l}\left\langle\phi_{k}|\mathbf{p}| \phi_{l}\right\rangle+\text { H.c. }\right] .
$$

In the tight binding approximation the matrix element for the momentum operator between the real atomic orbitals is given by [21]

$$
\left\langle\phi_{k}|\mathbf{p}| \phi_{l}\right\rangle=\frac{i m_{e}}{\hbar} \beta_{k l}\left(\mathbf{R}_{k}-\mathbf{R}_{l}\right) .
$$

Substituting (12) into (11) and taking the average over the ground state wave function in the external field yields exactly Eq. (9). This derivation completes the bond current formalism introduced by Pasquarello et al. [22]. The connection between dipole and anapole moments and the current can easily be obtained by substituting the appropriate vector potential into the classical formulas

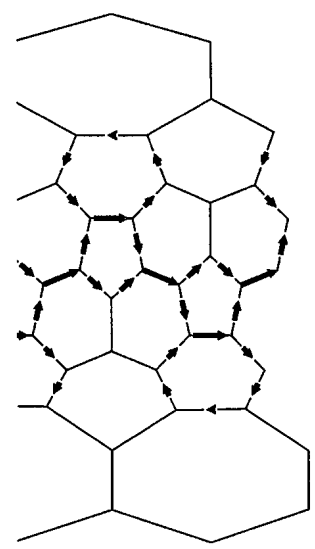

(a)

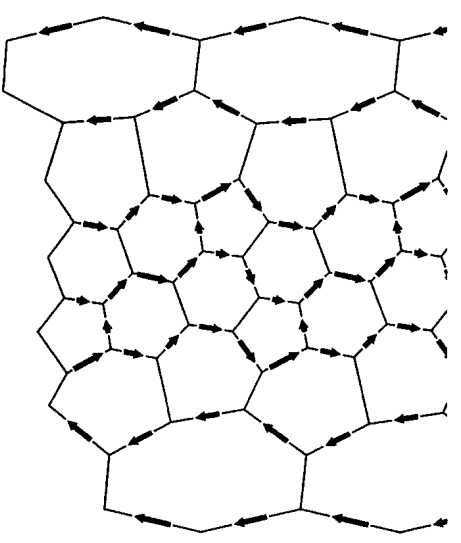

(b)
FIG. 3. Induced currents for the tori (a) and (b) under a uniform magnetic field, directed along the central symmetry axis. Only a section of the full toroidal surface is shown. Arrow lengths are proportional to current moduli.

for the energy of currents in the external magnetic field, followed by differentiation with respect to $\mathbf{B}$ or $\mathbf{B}^{\prime}$

$$
\begin{aligned}
\mathbf{m} & =\frac{1}{2 c} \int \mathbf{r} \times \mathbf{J} d v, \\
\mathbf{a} & =\frac{1}{3 c} \int \mathbf{r} \times(\mathbf{r} \times \mathbf{J}) d v .
\end{aligned}
$$

These volume integrals may be converted into line integrals along bonds, yielding

$$
\begin{aligned}
\mathbf{m} & =\frac{1}{2 c} \sum_{\langle i j\rangle} J_{i j} \mathbf{S}_{i j}, \\
\mathbf{a} & =\frac{1}{3 c} \sum_{\langle i j\rangle} J_{i j}\left(\mathbf{R}_{i}+\mathbf{R}_{j}\right) \times \mathbf{S}_{i j},
\end{aligned}
$$

where the sums run over distinct bonded pairs. These gauge-invariant current formulas yield exactly the same

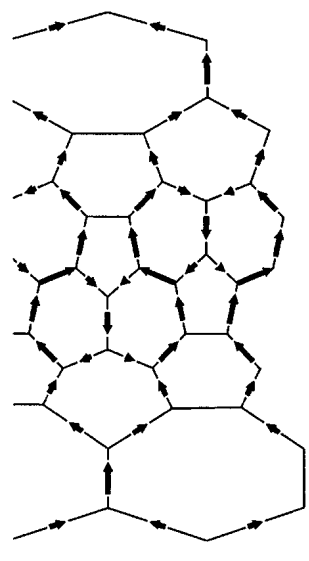

(a)

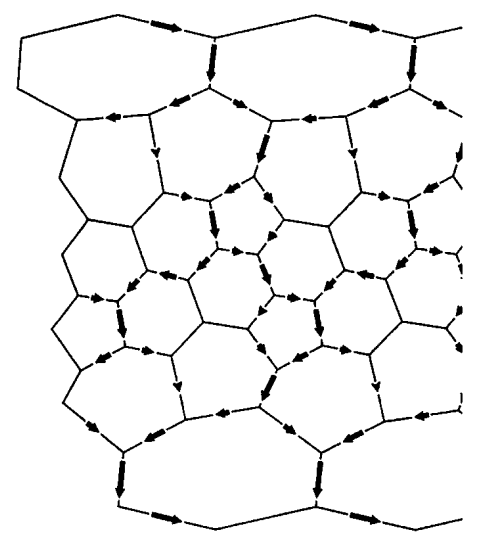

(b)
FIG. 4. Induced currents for the tori (a) and (b) under a rotor perturbation, directed along the central symmetry axis. 


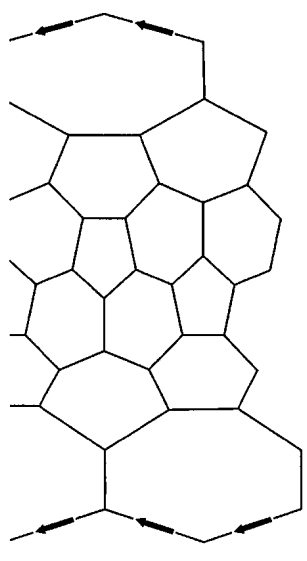

(a)

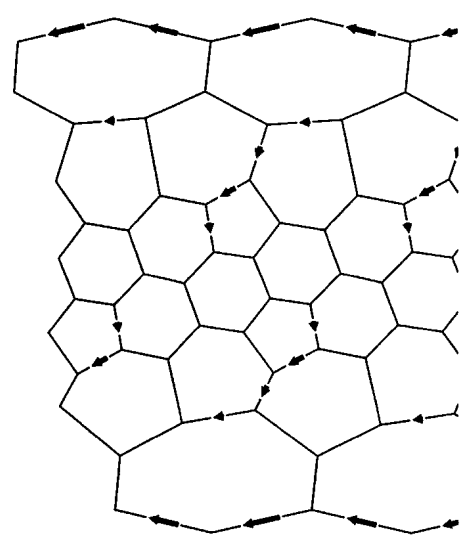

(b)
FIG. 5. Current states for the open-shell toroidal cations (a) and (b), provoked by application of an infinitesimal uniform field, showing the dominance in the frontier molecular orbital of circulation around the inner equator of the torus i.e., along the line of fusion of the heptagonal rings, and the mixed dipolar/ anapolar character of the flow in the chiral torus.

result as the operator formulation. The plots of the currents in Figs. 3-5 illustrate the outcome of the calculations presented in Table I. The induced currents were obtained by performing the calculations with nonzero axial magnetic field (Fig. 3) or rotor perturbation (Fig. 4). Clearly the axial field induces a current that circulates around the central hole of the torus, while the rotor field causes a current that whirls around in a perpendicular direction. For the monocations in degenerate states, permanent moments are found as illustrated in Fig. 5. In the achiral isomer this moment is entirely dipolar, but in the chiral isomer the current has a spiral pattern, consisting of a dipolar flow with an anapolar "leakage."

In conclusion, classical through-bond effects in carbon networks of higher topology can give rise to permanent and induced anapole moments and thereby bring a concept developed in the context of nuclear physics into the realm of molecular physics.

Financial support from the Belgian Government (Ministerie van het Wetenschapsbeleid), the Belgian National Science Foundation (NFWO), and Exeter University is gratefully acknowledged.
*On leave from the Institute of Chemistry, Academy of Sciences, Kishinev, Moldova.

[1] J. Liu, H. Dai, J. H. Hafner, D. T. Colbert, R. E. Smalley, S. J. Tans, and C. Dekker, Nature (London) 385, 780 (1997).

[2] A. Ceulemans and P. W. Fowler, J. Chem. Soc. Faraday Trans. 91, 3089 (1995).

[3] A. Ceulemans, M. Szopa, and P. W. Fowler, Europhys. Lett. 36, 645 (1996).

[4] Ya. B. Zel'dovich, Zh. Eksp. Teor. Fiz. 33, 1531 (1957) [JETP 6, 1184 (1957)].

[5] C. S. Wood, S. C. Bennett, D. Cho, B. P. Masterson, J. L. Roberts, C. E. Tanner, and C. E. Wieman, Science 275, 1759 (1997).

[6] M. G. Kozlov and L. N. Labzowsky, J. Phys. B 28, 1933 (1995).

[7] I. B. Khriplovich, Parity Nonconservation in Atomic Phenomena (Gordon and Breach, Philadelphia, PA, 1991).

[8] L. R. Hunter, Science 252, 73 (1991).

[9] A. M. Mårtensson-Pendrill, Atomic and Molecular Properties, edited by S. Wilson, Methods in Computational Chemistry Vol. 5 (Plenum, New York, 1992).

[10] R. R. Lewis and S. M. Blinder, Phys. Rev. A 52, 4439 (1995).

[11] I. B. Khriplovich and M.E. Pospelov, Z. Phys. D 17, 81 (1990).

[12] R. E. Raab, Mol. Phys. 29, 1323 (1975).

[13] A. Pasquarello, M. Schlüter, and R.C. Haddon, Science 257, 1660 (1992); R. C. Haddon, Nature (London) 378, 249 (1995).

[14] J. C. Greer, S. Itoh, and S. Ihara, Chem. Phys. Lett. 222, 621 (1994).

[15] A. J. Stone and D. J. Wales, Chem. Phys. Lett. 128, 501 (1986).

[16] F. London, J. Phys. Radium 8, 397 (1937).

[17] L. Salem, The Molecular Orbital Theory of Conjugated Systems (Benjamin, Reading, MA, 1972).

[18] V. Elser and R.C. Haddon, Phys. Rev. A 36, 4579 (1987).

[19] R. C. Haddon, Nature (London) 388, 31 (1997).

[20] L.D. Landau and E.M. Lifshitz, Quantum Mechanics (Pergamon, Oxford, 1975), 2nd ed.

[21] G. D. Mahan, Many-Particle Physics (Plenum, New York, 1981).

[22] A. Pasquarello, M. Schlüter, and R.C. Haddon, Phys. Rev. A 47, 1783 (1993). 